\title{
Call Blocking Probabilities for Poisson Traffic Under the Multiple Fractional Channel Reservation Policy
}

\author{
I. D. Moscholios*, V. G. Vassilakis** and M. D. Logothetis*** \\ *Dept. of Informatics \& Telecommunications, University of Peloponnese, 22100 Tripolis, Greece. \\ ** School of Computing, Engineering \& Mathematics, University of Brighton, BN2 4NT Brighton, U.K. \\ ***WCL, Dept. of Electrical \& Computer Engineering, University of Patras, 26504 Patras, Greece. \\ E-mails: idm@uop.gr, v.vasilakis@brighton.ac.uk, mlogo@upatras.gr
}

\begin{abstract}
In this paper, we study the performance of the Multiple Fractional Channel Reservation (MFCR) policy, which is a bandwidth reservation policy that allows the reservation of real (not integer) number of channels in order to favor calls of high channel (bandwidth) requirements. We consider a link of fixed capacity that accommodates Poisson arriving calls of different service-classes with different bandwidth-per-call requirements. Calls compete for the available bandwidth under the MFCR policy. To determine call blocking probabilities, we propose approximate but recursive formulas based on the notion of reserve transition rates. The accuracy of the proposed method is verified through simulation.
\end{abstract}

Keywords - call blocking; bandwidth reservation; recursive formula; Markov; product-form.

\section{INTRODUCTION}

Multirate loss models play an important role in the performance modelling and evaluation of contemporary networks that service calls from different service-classes with different bandwidth-per-call requirements. Depending on the selected bandwidth allocation policy, which defines the way system resources are shared among service-classes, a Call Admission Controller (CAC) usually treats some service-class calls differently from others; for instance, new arriving calls may be treated differently than in-service calls, which may alter their bandwidth. For this purpose, we need an appropriate bandwidth allocation policy.

The Complete Sharing (CS) policy (also known as fullavailability group [1]) is the simplest bandwidth allocation policy [2]. In the CS policy, a new call is accepted in the system whenever the available system's bandwidth is equal to or greater than the call's required bandwidth, i.e., the only constraint of the CS policy is the system's capacity. The main drawback of the CS policy is that it cannot provide a certain QoS to calls of a serviceclass. A simple policy that achieves this target and is broadly applicable in wired, wireless and satellite networks (e.g., [3]-[8]) is the Threshold (TH) policy. In the TH policy, a new call of a service-class is accepted in the system if the number of in-service calls of that service-class does not exceed a predefined threshold.

Another policy whereby QoS can be guaranteed to new calls is the Bandwidth Reservation (BR) policy (also known in the literature as guard channel policy [9]). In the BR policy, an integer number of bandwidth units (b.u.) or channels is reserved to benefit calls of high bandwidth requirements. The BR policy achieves Call Blocking Probabilities (CBP) equalization among service-classes at the cost of substantially increasing the CBP of calls with lower bandwidth requirements (e.g., [10]-[11]). The fact that the BR policy has been broadly applied in wired (e.g., [12]-[19]), wireless (e.g., [20][26]) and optical networks (e.g., [27]-[28]) evinces its importance in call admission control.

In this paper, we focus on an extension of the BR policy, namely the multiple fractional channel reservation (MFCR) policy [29], applied in a single link of fixed capacity that accommodates Poisson arriving calls of different service-classes with different bandwidth-percall requirements. The link is modelled as a multirate loss system. The MFCR policy extends the BR policy by allowing the reservation of real number of channels (or b.u.). More precisely, in the MFCR policy, real number of channels, $t_{r, k}$, are reserved to benefit calls from all service-classes apart from service-class $k$ calls. The reservation of real number of channels is achieved since $\left\lfloor t_{r, k}\right\rfloor+1$ channels are reserved with probability $t_{r, k}-\left\lfloor t_{r, k}\right\rfloor$, while $\left\lfloor t_{r, k}\right\rfloor$ channels are reserved with probability $1-\left(t_{r, k}-\left\lfloor t_{r, k}\right\rfloor\right)$, where $\left\lfloor t_{r, k}\right\rfloor$ is the largest integer not exceeding $t_{r, k}$. The application of the MFCR policy destroys local balance between adjacent states and therefore it leads to non-reversible continuous time Markov chains, which have no product form solution for the steady-state distribution. This leads to an approximate but recursive formula for the determination of the link occupancy distribution and consequently the CBP calculation [29]. The formula of [29] resembles the classical Roberts formula proposed for a multirate loss system that accommodates Poisson arriving calls under the BR policy [12].

To improve the accuracy of the CBP calculation of [29], compared to simulation results, we consider a method that takes into account the Reverse Transition Rates (RTR), which may occur for a service-class inside its reservation space (see Section III). The RTR method has been initially proposed in [13] in order to improve the accuracy of Roberts formula in the case of the BR policy. The RTR method does improve the accuracy of the 
analytical CBP results in various teletraffic loss models, especially when CBP equalization is required among service-classes (see e.g., [30]-[31]).

This paper is organized as follows: In Section II, we review the model of [29]. In Section III, we present the RTR method in the case of the MFCR policy and provide formulas for the calculation of the link occupancy distribution and CBP. In Section IV, we present analytical CBP results both for the proposed model (MFCR/RTR) and the model of [29]. Comparison of the analytical results with simulation results shows that the RTR method achieves better accuracy compared to [29]. We conclude in Section V.

\section{THE MFCR POLICY}

Consider a single link of capacity $C$ channels (or b.u.) that accommodates calls of $K$ different service-classes under the MFCR policy. A call of service class $k$ ( $k$ $=1, \ldots, K)$ follows a Poisson process with arrival rate $\lambda_{k}$, requests $b_{k}$ channels and has an MFCR parameter $t_{r, k}$ that expresses the reserved real number of channels used to benefit calls of all other service-classes except from service-class $k$. The reservation of $t_{r, k}$ channels is achieved because $\left\lfloor t_{r, k}\right\rfloor+1$ channels are reserved with probability $t_{r, k}-\left\lfloor t_{r, k}\right\rfloor$ while $\left\lfloor t_{r, k}\right\rfloor$ channels are reserved with probability $1-\left(t_{r, k}-\left\lfloor t_{r, k}\right\rfloor\right)$. As an example, calls of service-class $k$ may have an MFCR parameter of $t_{r, k}=1.3$ channels. The reservation of 1.3 channels is achieved by assuming that $\lfloor 1.3\rfloor+1=2$ channels are reserved with probability $1.3-\lfloor 1.3\rfloor=0.3$ while $\lfloor 1.3\rfloor=1$ channels is reserved with probability $1-(1.3-\lfloor 1.3\rfloor)=0.7$.

Let $j$ be the occupied link bandwidth $(j=0,1 \ldots, C)$ when a new service-class $k$ call arrives in the link. Then, we consider the following cases: a) if the available link bandwidth $(C-j)$ minus the MFCR parameter $\left\lfloor t_{r, k}\right\rfloor$ is higher than the required $b_{k}$ channels i.e., if $C-j-\left\lfloor t_{r, k}\right\rfloor>b_{k}$, then the new call is accepted in the system, b) if $C-j-\left\lfloor t_{r, k}\right\rfloor=b_{k}$, then the new call is accepted in the system with probability $1-\left(t_{r, k}-\left\lfloor t_{r, k}\right\rfloor\right)$ and c) if $C-j-\left\lfloor t_{r, k}\right\rfloor<b_{k}$, then there is no available link bandwidth and the new call is blocked and lost without further affecting the system. An accepted call (cases (a) and (b)) remains in the system for an exponentially distributed service time with mean $\mu_{k}^{-1}$.

The determination of the link occupancy distribution, $G(j)$, in the MFCR is based on the following approximate but recursive formula [29]:

$$
G(j)=\left\{\begin{array}{c}
1 \text { for } j=0 \\
\frac{1}{j} \sum_{k=1}^{K} a_{k} D_{k}\left(j-b_{k}\right) G\left(j-b_{k}\right) \text { for } j=1, \ldots, C \\
\text { Ootherwise }
\end{array}\right.
$$

where: $a_{k}=\lambda_{k} \mu_{k}^{-1}$ is the total offered traffic-load of service-class $k$ calls (in erl) and

$$
D_{k}\left(j-b_{k}\right)=\left\{\begin{array}{l}
b_{k} \text { for } j<C-\left\lfloor t_{r, k}\right\rfloor \\
\left(1-\left(t_{r, k}-\left\lfloor t_{r, k}\right\rfloor\right)\right) b_{k} \text { for } j=C-\left\lfloor t_{r, k}\right\rfloor \\
0 \text { for } j>C-\left\lfloor t_{r, k}\right\rfloor
\end{array}\right.
$$

Having determined $G(j)$ 's we can calculate the CBP of service-class $k$ calls, $B_{k}$, as follows [29]:

$$
\begin{aligned}
& B_{k}=\sum_{j=C-b_{k}-\left\lfloor t_{r, k}\right\rfloor+1}^{C} G^{-1} G(j) \\
& +\left(t_{r, k}-\left\lfloor t_{r, k}\right\rfloor\right) G^{-1} G\left(C-b_{k}-\left\lfloor t_{r, k}\right\rfloor\right)
\end{aligned}
$$

where: $G=\sum_{j=0}^{C} G(j)$ is the normalization constant.

In addition, we can calculate performance metrics such as the link utilization, $U$, via (4), or the average number of service-class $k$ calls in state $j, y_{k}(j)$, via (5):

$$
\begin{aligned}
& U=\sum_{j=1}^{C} j G^{-1} G(j) \\
& y_{k}(j)=\left\{\begin{array}{l}
\frac{a_{k} G\left(j-b_{k}\right)}{G(j)} \text { for } j<C-\left\lfloor t_{r, k}\right\rfloor \\
\frac{a_{k}\left(1-\left(t_{r, k}-\left\lfloor t_{r, k}\right\rfloor\right)\right) G\left(j-b_{k}\right)}{G(j)} \text { for } j=C-\left\lfloor t_{r, k}\right\rfloor \\
0 \text { for } j>C-\left\lfloor t_{r, k}\right\rfloor
\end{array}\right.
\end{aligned}
$$

Note that (5) is the basis for the proof of (1) and implies that the average number of calls in state $j, y_{k}(j)$, is negligible in the part of the reservation space of serviceclass $k$ denoted by the states: $j=C-\left\lfloor t_{r, k}\right\rfloor+1, \ldots, C$. However, as we will see in the next section, there exist RTR from such states $j$, a fact that can improve the accuracy of the CBP calculation.

In the case of the classical BR policy (where an integer number of channels is reserved), the link occupancy distribution is determined by (1), the link utilization by (4) while (2), (3) and (5) take the form of (6), (7) and (8), respectively [13]:

$$
\begin{aligned}
& D_{k}\left(j-b_{k}\right)= \begin{cases}b_{k} & \text { for } j \leq C-\left\lfloor t_{r, k}\right\rfloor \\
0 & \text { for } j>C-\left\lfloor t_{r, k}\right\rfloor\end{cases} \\
& B_{k}=\sum_{j=C-b_{k}-\left\lfloor t_{r, k}\right\rfloor+1}^{C} G^{-1} G(j) \\
& y_{k}(j)= \begin{cases}\frac{a_{k} G\left(j-b_{k}\right)}{G(j)} \text { for } j \leq C-\left\lfloor t_{r, k}\right\rfloor \\
0 \text { for } j>C-\left\lfloor t_{r, k}\right\rfloor\end{cases}
\end{aligned}
$$

In the case of the CS policy (all BR parameters are set to zero), the link occupancy distribution is determined by the classical Kaufman-Roberts recursion (9), CBP by (10) and the values of $y_{k}(j)$ by (11), [32]-[33]: 


$$
\begin{aligned}
& G(j)=\left\{\begin{array}{c}
1 \text { for } j=0 \\
\frac{1}{j} \sum_{k=1}^{K} a_{k} b_{k} G\left(j-b_{k}\right) \text { for } j=1, \ldots, C \\
\text { 0otherwise }
\end{array}\right. \\
& B_{k}=\sum_{j=C-b_{k}+1}^{C} G^{-1} G(j) \\
& y_{k}(j)=\left\{\begin{array}{c}
\frac{a_{k} G\left(j-b_{k}\right)}{G(j)} \text { for } j \leq C \\
0 \quad \text { for } j>C
\end{array}\right.
\end{aligned}
$$

\section{THE RTR METHOD IN THE MFCR POLICY}

To illustrate the RTR method, consider a link with capacity $C=3$ channels that accommodates calls of two service-classes. Let the corresponding offered traffic loads and bandwidth requirements be $a_{1}, a_{2}, b_{1}=1$ and $b_{2}=2$ channels, respectively. Calls of the $1^{\text {st }}$ service-class have an MFCR parameter of $t_{r, 1}=1.3$ channels.

Fig. 1 presents the one-dimensional Markov chain for this system. Each state corresponds to the occupied link bandwidth $j=0,1,2,3$.

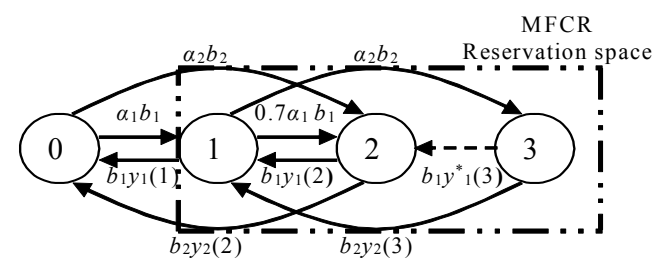

Figure 1. One-dimensional Markov chain of the example.

Based on (5), the average number of $1^{\text {st }}$ service-class calls is zero in state $j=3$, i.e., $y_{1}(3)=0$. Assume now that a call of the $2^{\text {nd }}$ service-class arrives in the system and finds one call from the $1^{\text {st }}$ service-class under service (i.e., at the time of arrival the system state is $j=1$ ). The new call will be accepted in the link and the new state is $j=3$. Thus, in state $j=3$ there is still the $1^{\text {st }}$ service-class call under service. This means that there should exist a RTR $b_{1} y_{1}^{*}(3)$ from state $j=3$ towards $j=2$, where $y_{1}^{*}(3)$ is the average number of calls of the $1^{\text {st }}$ service-class in state $j=3$ which should be calculated. This modification is presented in Fig. 1 with the dashed arrow.

To determine the values of the modified RTR, $y_{k}^{*}(j)$ we propose the following approximate formula:

$y_{k}(j) \equiv y_{k}^{*}(j)=\left\{\begin{array}{l}\frac{a_{k} G\left(j-b_{k}\right)}{G(j)}, \text { for } j<C-\left\lfloor t_{r, k}\right\rfloor \\ \sum_{i=1, i \neq k}^{K} y_{k}^{*}\left(j-b_{i}\right) w_{k, i}(j), \text { for } j \geq C-\left\lfloor t_{r, k}\right\rfloor\end{array}\right.$

where: $G(j)$ 's will be determined by (9) (the accurate Kaufman-Roberts formula) for $j<C-\left\lfloor t_{r, k}\right\rfloor$ while $w_{k, i}(j)$ is a weight factor given by the following approximate formula:

$$
w_{k, i}(j)=\left\{\begin{array}{l}
\frac{a_{i} b_{i}}{\sum_{x=1, x \neq k}^{K} a_{x} b_{x}}, \text { for } j<C-\left\lfloor t_{r, i}\right\rfloor \\
\frac{\left(1-\left(t_{r, i}-\left\lfloor t_{r, i}\right\rfloor\right)\right) a_{i} b_{i}}{\sum_{x=1, x \neq k}^{K} a_{x} b_{x}}, \text { for } j=C-\left\lfloor t_{r, i}\right\rfloor
\end{array}\right.
$$

where:

$a_{x} b_{x}=\left\{\begin{array}{l}a_{x} b_{x}, \text { for } j<C-\left\lfloor t_{r, x}\right\rfloor \\ \left(1-\left(t_{r, x}-\left\lfloor t_{r, x}\right\rfloor\right)\right) a_{x} b_{x}, \text { for } j=C-\left\lfloor t_{r, x}\right\rfloor\end{array}\right.$

The weight $w_{k, i}(j)$ determines the proportion of the RTR $y_{k}^{*}(j)$ that is transferred in state $j$ by a new serviceclass $i$ call (other than service-class $k$ ). Although the system cannot be in state $j$ due to an arriving call of service-class $k$ (because of the MFCR policy), the system can be in state $j$ due to arriving calls of other serviceclasses. Thus, when the system is transferred to state $j$ by a service-class $i$ call, this call transfers to state $j$ the population of service-class $k$, too. Therefore, the assumption that the average number of calls is positive even in a prohibitive state of a service-class is more realistic compared to the assumption that it is negligible.

Having calculated $y_{k}^{*}(j)$ we continue by determining the modified occupancy distribution:

$$
G(j)=\left\{\begin{array}{c}
1 \text { for } j=0 \\
\frac{1}{j^{*}} \sum_{k=1}^{K} a_{k} D_{k}\left(j-b_{k}\right) G\left(j-b_{k}\right) \text { for } j=1, \ldots, C \\
\text { Ootherwise }
\end{array}\right.
$$

where: $j^{*}=\sum_{k=1}^{K} b_{k} y_{k}^{*}(j)$ and $D_{k}\left(j-b_{k}\right)$ is given by (2).

The philosophy behind the proposed RTR method is that the approximated reversible Markov chain of the MFCR policy is kept, but the value of each state $j$ of the prohibited state space is now substituted by $j^{*}$.

As far as the CBP are concerned, they can be determined by (3).

\section{NumERICAL EXAMPLES - EVAlUATION}

In this section, we present an application example and provide analytical CBP results of the proposed method (MFCR/RTR) and the MFCR policy of [29]. As a reference we also include analytical CBP results for the CS policy [32]-[33]. In addition, we present simulation results of the MFCR policy, in order to show that the proposed method gives CBP results closer to the simulation results. The latter are based on the Simscript III simulation language [34] and are mean values of 7 runs. As far as the reliability ranges are concerned (confidence intervals of $95 \%$ calculated via the t-Student distribution) they are less than two order of magnitude, and therefore we do not present them in the following figures. All simulation runs are based on the generation 
of four million calls per run. To account for a warm-up period, the first $5 \%$ of these generated calls are not considered in the CBP results.

As an application example, consider a link of capacity $C=60$ channels, that accommodates calls of three service-classes, with the traffic characteristics of Table 1.

Table 1: Service-classes - Traffic characteristics

\begin{tabular}{|c|c|c|c|}
\hline $\begin{array}{c}\text { Service- } \\
\text { class }\end{array}$ & $\begin{array}{c}\text { Traffic-load } \\
(\text { erl) }\end{array}$ & $\begin{array}{c}\text { Bandwidth per } \\
\text { call (channels) }\end{array}$ & $\begin{array}{c}\text { MFCR parameter } \\
\text { (channels) }\end{array}$ \\
\hline $1^{\text {st }}$ & $a_{1}=1.0$ & $b_{1}=1$ & $t_{r, 1}=9.4$ \\
\hline $2^{\text {nd }}$ & $a_{2}=1.0$ & $b_{2}=5$ & $t_{r, 2}=5.3$ \\
\hline $3^{\text {rd }}$ & $a_{3}=1.0$ & $b_{3}=10$ & $t_{r, 3}=0$ \\
\hline
\end{tabular}

Concerning the MFCR parameter of the $1^{\text {st }}$ serviceclass, the reservation of 9.4 channels is achieved by assuming that $\lfloor 9.4\rfloor+1=10$ channels are reserved with probability 0.4 while $\lfloor 9.4\rfloor=9$ channels are reserved with probability 0.6 . Similarly, the MFCR parameter of 5.3 channels for the $2^{\text {nd }}$ service-class is achieved by assuming that $\lfloor 5.3\rfloor+1=6$ channels are reserved with probability 0.3 while $\lfloor 5.3\rfloor=5$ channels are reserved with probability 0.7 .

In the $x$-axis of Figs 2-4 the offered traffic load of the $1^{\text {st }}, 2^{\text {nd }}$ and $3^{\text {rd }}$ service-class increases in steps of $0.5,0.20$ and 0.1 erl, respectively. So, point 1 is: $\left(a_{1}, a_{2}, a_{3}\right)=(1.0$, $1.0,1.0)$ while point 11 is: $\left(\alpha_{1}, \alpha_{2}, \alpha_{3}\right)=(6.0,3.0,2.0)$.

In Figs. 2-4, we present the analytical CBP results of the MFCR/RTR method, the MFCR and the CS policies together with the MFCR simulation results, for each service-class, respectively. All figures show that the analytical results of the proposed MFCR/RTR are closer to the corresponding simulation results compared to the results obtained by the MFCR policy of [29]. A large amount of examples reveals that the MFCR/RTR method provides $\mathrm{CBP}$ results closer to the simulation results, especially when the MFCR parameters are large (e.g., when CBP tend to be equal among calls of serviceclasses). This is expected, since large values of the MFCR parameters lead to large MFCR reservation spaces and therefore to many states $j$ (that belong to the reservation space) whereby $y_{k}^{*}(j)$ 's are calculated.

\section{CONCLUSION}

We propose a method based on the RTR for the CBP calculation in a multirate teletraffic loss model of a single link that accommodates Poisson arriving calls under the MFCR policy. The proposed method is based on the fact that RTR do appear in the reservation space of each service-class. The CBP results obtained by the proposed method compared to simulation results show that the proposed method improves the calculation's accuracy. As a future work, we intend to study this method in the case of quasi-random traffic (traffic generated by a finite number of users). In addition, we will extend the proposed method in the case of a network of links with different capacities. To analyze such a system, the reduced load approximation could be used [2]. The latter provides highly satisfactory results compared to simulation.

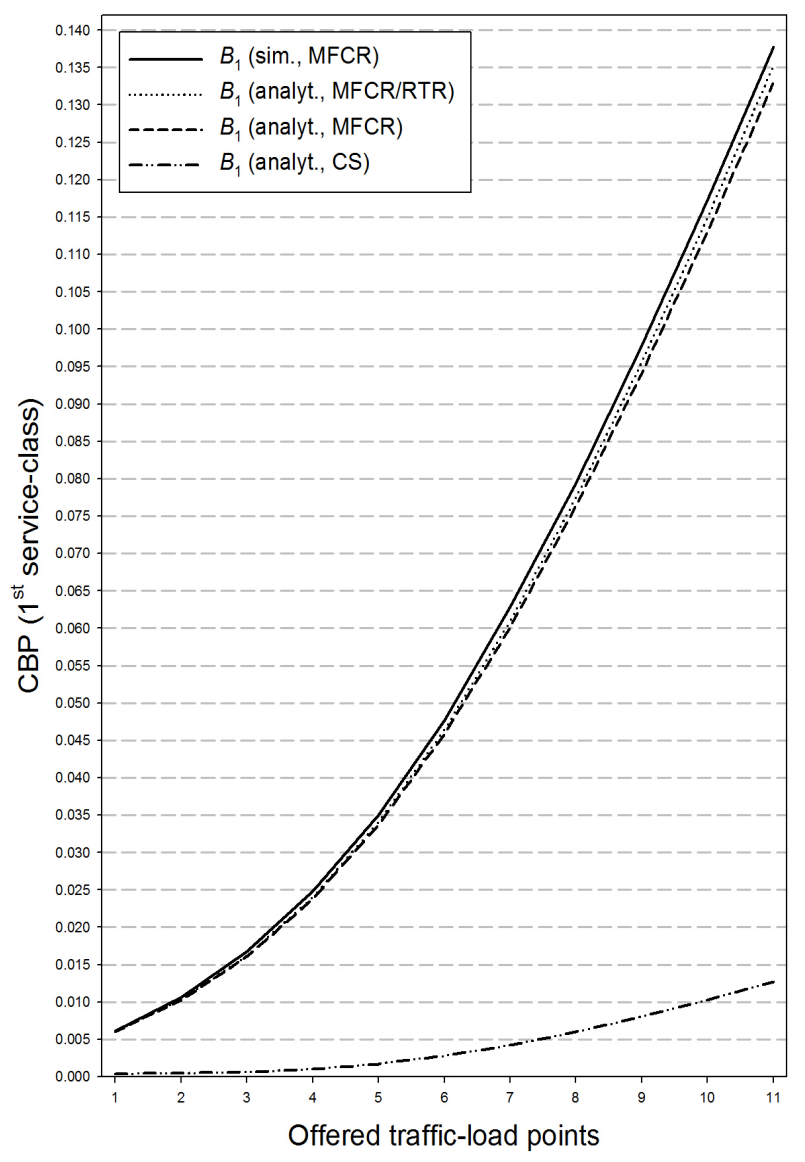

Figure 2. $\mathrm{CBP}-1^{\text {st }}$ service-class.

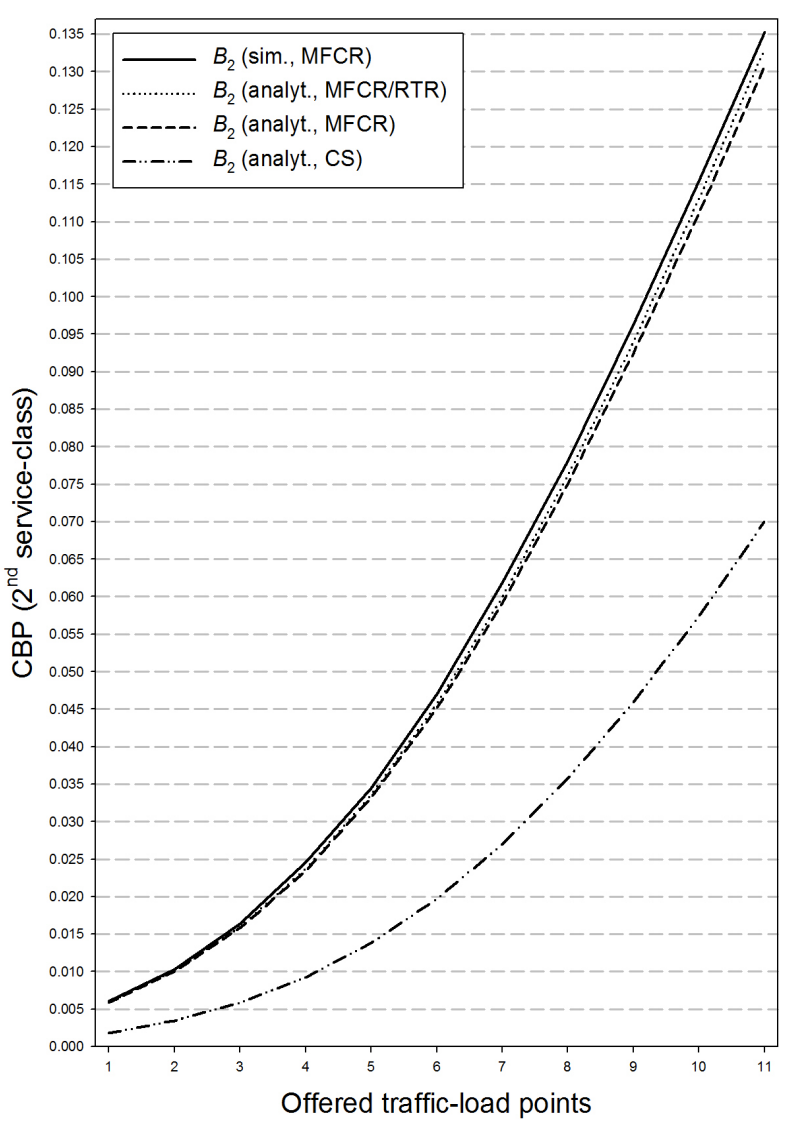

Figure 3. $\mathrm{CBP}-2^{\text {nd }}$ service-class. 


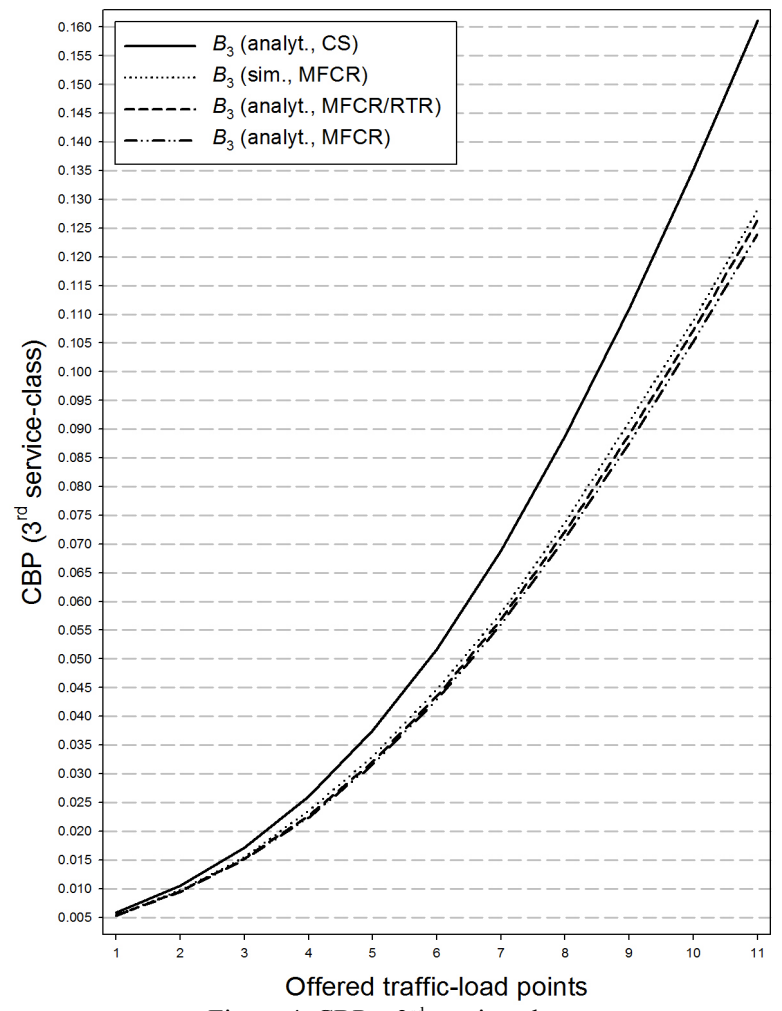

Figure 4. CBP $-3^{\text {rd }}$ service-class.

\section{REFERENCES}

1. M. Stasiak, M. Glabowski, A.Wisniewski, and P. Zwierzykowski, Modeling and Dimensioning of Mobile Networks, Wiley \& Sons, 2011.

2. K. Ross, Multiservice Loss Models for Broadband Telecommunication Networks, Springer, 1995.

3. J. Ni, D. Tsang, S. Tatikonda and B. Bensaou, "Optimal and Structured Call Admission Control Policies for Resource-Sharing Systems", IEEE Trans. Commun., 55 (1), pp. 158-170, Jan. 2007.

4. I. Moscholios, M. Logothetis, J. Vardakas and A. Boucouvalas, "Performance Metrics of a Multirate Resource Sharing Teletraffic Model with Finite Sources under both the Threshold and Bandwidth Reservation Policies”, IET Networks, 4 (3), pp. 195 208, May 2015.

5. I. Moscholios, M. Logothetis, A. Boucouvalas and V. Vassilakis, "An Erlang Multirate Loss Model Supporting Elastic Traffic under the Threshold Policy", Proc. IEEE ICC, London, U.K., June 2015.

6. A. Al Daoud, M. Alanyali, and D. Starobinski, "Pricing strategies for spectrum lease in secondary markets", IEEE/ACM Trans.Netw., 18 (2), pp. 462-475, Apr. 2010.

7. X. Y. Yu and H. B. Zhu, "An efficient method for loss performance modeling of hierarchical heterogeneous wireless networks", Int. Journal of Commun. Systems, 27 (6), pp. 956-968, June 2014.

8. Z. Wang, P. Mathiopoulos, and R. Schober, "Channel partitioning policies for multi-class traffic in LEO-MSS”, IEEE Trans. Aerosp. Electron. Syst., 45 (4), pp. 1320 - 1334, Oct. 2009.

9. R. Ramjee, D. Towsley and R. Nagarajan, "On optimal call admission control in cellular networks", Wireless Networks, 3 (1), pp. 29-41, March 1997.

10. M. Glabowski, A. Kaliszan and M. Stasiak, "Asymmetric convolution algorithm for blocking probability calculation in fullavailability group with bandwidth reservation", IET Circuits, Devices \& Systems, 2 (1), pp.87-94, Feb. 2008.

11. I. Moscholios, J. Vardakas, M. Logothetis and A. Boucouvalas, "A Batched Poisson Multirate Loss Model Supporting Elastic Traffic under the Bandwidth Reservation Policy", Proc. IEEE ICC, Kyoto, Japan, June 2011.

12. J. Roberts, "Teletraffic models for the Telecom 1 Integrated Services Network", Proc. 10th ITC, paper 1.1-2, Montreal 1983.
13. M. Stasiak, M. Glabowski, "A simple approximation of the link model with reservation by a one-dimensional Markov chain", Performance Evaluation, 41 (2-3), pp. 195-208, July 2000.

14. M. Glabowski, "Modelling of state-dependent multirate systems carrying BPP traffic", Annals of Telecommunications, 63 (7), pp. 393-407, August 2008.

15. I. Moscholios, J. Vardakas, M. Logothetis and A. Boucouvalas, "QoS Guarantee in a Batched Poisson Multirate Loss Model Supporting Elastic and Adaptive Traffic", Proc. IEEE ICC, Ottawa, Canada, June 2012.

16. M. Glabowski, M. Sobieraj and M. Stasiak, "Modelling Limitedavailability Systems with Multi-service Sources and Bandwidth Reservation", Proc. 8th AICT, Stuttgart, Germany, June 2012.

17. I. Moscholios, V. Vassilakis, M. Logothetis and M. Koukias, "QoS Equalization in a Multirate Loss Model of Elastic and Adaptive Traffic with Retrials", Proc. 5th EMERGING, Porto, Portugal, October 2013.

18. I. Moscholios, J. Vardakas, M. Logothetis and M. Koukias, "A Quasi-random Multirate Loss Model supporting Elastic and Adaptive Traffic under the Bandwidth Reservation Policy", Int. Journal on Advances in Networks and Services, 6 (3\&4), pp. 163174, December 2013.

19. L. Brewka, V. Iversen and G. Kardaras, "Integrated service resource reservation using queueing networks", IET Networks, 3 (1), pp. 16-21, March 2014.

20. C. Oliveira, J. Kim, and T. Suda, "An adaptive bandwidth reservation scheme for high-speed multimedia wireless networks", IEEE J. Select. Areas Commun., 16 (6), pp. 858-874, Aug. 1998.

21. I. Moscholios, M. Logothetis and M. Koukias, "A StateDependent Multi-Rate Loss Model of Finite Sources with QoS Guarantee for Wireless Networks", Mediterranean Journal of Computers and Networks, 2 (1), pp. 10-20, Jan. 2006.

22. M. Glabowski, M. Sobieraj and M. Stasiak, "Evaluation of traffic characteristics of UMTS with bandwidth reservation and handoff mechanism”, Proc. ICT-MICC, Penang, Malaysia, May 2007.

23. G. Raskutti, A. Zalesky, E. Wong and M. Zukerman, "Enhanced Blocking Probability Evaluation Method for Circuit-Switched Trunk Reservation Networks," IEEE Commun. Letters, 11 (6), pp.543-545, June 2007.

24. M. Stasiak, P. Zwierzykowski, D. Parniewicz, "Modelling of the WCDMA Interface in the UMTS Network with Soft Handoff Mechanism", Proc. IEEE Globecom, Honolulu, Nov. 2009.

25. M. Stasiak, D. Parniewicz and P. Zwierzykowski, "Traffic Engineering for Multicast Connections in Multiservice Cellular Network", IEEE Transactions on Industrial Informatics, 9 (1), pp. $262-270$, Feb. 2013.

26. I. Moscholios, G. Kallos, M. Katsiva, V. Vassilakis and M Logothetis, "Call Blocking Probabilities in a W-CDMA cell with interference cancellation and bandwidth reservation", Proc. IEICE ICTF, Poznan, Poland, May 2014.

27. J. Vardakas, I. Moscholios, M. Logothetis and V. Stylianakis, "On Code reservation in Multi-rate OCDMA Passive Optical Networks", Proc. CSNDSP, Poznan, Poland, July 2012.

28. F. Callegati, et al., "Trunk reservation for fair utilization in flexible optical networks", IEEE Commun. Letters, 18 (5), pp. 889-892, May 2014.

29. F. Cruz-Pérez, J. Vázquez-Ávila and L. Ortigoza-Guerrero, "Recurrent formulas for the multiple fractional channel reservation strategy in multi-service mobile cellular networks", IEEE Commun. Letters, 8 (10), pp. 629-631, Oct. 2004.

30. I. Moscholios, M. Logothetis and T. Liokos, "QoS Equalization in the Connection Dependent Threshold Model", Proc. CSNDSP, Staffordshire, U.K, July 2002.

31. I. Moscholios and M. Logothetis, "The Erlang Multirate Loss Model with Batched Poisson Arrival Processes under the Bandwidth Reservation Policy", Computer Communications, 33 (1), pp. S167-S179, Nov. 2010.

32. J. Kaufman, "Blocking in a shared resource environment", IEEE Trans. Commun., 29 (10), pp. 1474-1481, Oct. 1981.

33. J. Roberts, "A service system with heterogeneous user requirements", in Performance of Data Commun. systems and their applications, North Holland, Amsterdam, pp.423-431, 1981.

34. Simscript III, http://www.simscript.com/ (accessed: May 2016). 